\title{
Bird's Eye View Transformation Technique in Photogrammetric Problem of Object Size Measuring at Low-altitude Photography
}

\author{
Ivan S. Kholopov \\ Ryazan State Radio Engineering University (RSREU) \\ Ryazan, Russia \\ kholopov.i.s@rsreu.ru
}

\begin{abstract}
The paper considers the solution of the photogrammetric problem of measuring the planar objects dimensions with projective distortions that are characteristic for low-altitude photography. It is shown that a bird's eye view transformation technique allows estimating the area occupied by planar objects with a relative error of up to $5 \%$.
\end{abstract}

Keywords-projective distortion; bird's eye view transormation; homography matrix; quaternion; three-axis accelerometer; camera calibration

\section{INTRODUCTION}

Projective distortion is a type of geometric distortion that typical for low-altitude photo and video shooting, which violate the form and the true relative position of objects on the image frame. Correction of projective distortion is performed when drawing up plans or maps of the terrain, based on the results of optical-electronic aerial photography [1], for combining images taken from different angles [2-4], the shooting of texts [5] and rectangular planar patterns (such as maps, pictures or posters) [6], vehicle surround monitoring [7] and parking assistance [8], lane line segmentation and detection [9], etc. In the photogrammetric problem of the object size estimation, such type of distortion leads to incorrect measuring results.

\section{Projective Transormations AND HomograPhy MatriX}

Projective linear transformations can be presented as:

$$
\mathbf{m}_{2}=\mathbf{H} \mathbf{m}_{1} \text {, }
$$

where $\mathbf{m}_{1}$ and $\mathbf{m}_{2}$ are, respectively, the homogeneous pixel coordinates of the image before and after the transformation, and

$$
\mathbf{H}=\left[\begin{array}{ccc}
h_{11} & h_{12} & h_{13} \\
h_{21} & h_{22} & h_{23} \\
h_{31} & h_{32} & 1
\end{array}\right]
$$

is a homography matrix [10].

The homography matrix for a projective transformation can be represented as the product of matrices of affine $\mathbf{H}_{\mathrm{a}}$ and proper projective $\mathbf{H}_{\mathrm{p}}$ transformations [11]:

$$
\mathbf{H}=\mathbf{H}_{\mathrm{a}} \mathbf{H}_{\mathrm{p}},
$$

where

$$
\mathbf{H}_{\mathrm{p}}=\left[\begin{array}{ccc}
1 & 0 & 0 \\
0 & 1 & 0 \\
h_{31} & h_{32} & 1
\end{array}\right], \mathbf{H}_{\mathrm{a}}=\mathbf{H}_{\mathrm{t}} \mathbf{H}_{\mathrm{r}} \mathbf{H}_{\mathrm{s}}
$$

and

$$
\mathbf{H}_{\mathrm{s}}=\left[\begin{array}{ccc}
s_{u} & 0 & 0 \\
0 & s_{v} & 0 \\
0 & 0 & 1
\end{array}\right], \mathbf{H}_{\mathrm{r}}=\left[\begin{array}{ccc}
c & -s & 0 \\
s & c & 0 \\
0 & 0 & 1
\end{array}\right], \mathbf{H}_{\mathrm{t}}=\left[\begin{array}{ccc}
1 & 0 & \Delta u \\
0 & 1 & \Delta v \\
0 & 0 & 1
\end{array}\right]
$$

are matrices that specify the transformations of scaling, rotation and translation; $c=\cos \varphi, s=\sin \varphi, \varphi-$ the rotation angle counted in the counterclockwise direction, and $s_{u}, s_{v}$ and $\Delta u, \Delta v$ are the scale factors and translation values at the horizontal and vertical direction, respectively.

The problem of projective image distortion correction usually consists in estimating the matrix that is inverse to the homography matrix:

$$
\mathbf{m}_{1}=\mathbf{H}^{-1} \mathbf{m}_{2} \text {. }
$$

Depending on the presence or absence of a priori information about the parameters of the projective transformation and the observed objects, two main approaches of the homography matrix estimation can be applied for finding the solution of the projective distortion compensation problem. 


\section{A. Homography Matrix Estimation for Images of a Priori Known Form Objects}

If a priori it is known that the contour of the object in image is approximated by a convex $n$-gon, $n \geq 4$, and occupies the greater part of the frame (Fig. 1), then its vertices pixel coordinates can be estimated using Hough or Radon transforms [12]. Next, the desired vertices coordinates in the image without projective distortion $\left(u_{2}, v_{2}\right)$ are used for constructing (by stacking) a system of linear equations for the classical homography matrix estimation procedure by $n$ points' correspondences [10]:

$$
\mathbf{T}_{2 n \times 9} \mathbf{h}=\mathbf{0},
$$

where $\mathbf{h}=\left[h_{11}, h_{12}, h_{13}, h_{21}, h_{22}, h_{23}, h_{31}, h_{32}, 1\right]^{\mathrm{T}}$ and

$$
\mathbf{T}_{2 \times 9}=\left[\begin{array}{ccccccccc}
u_{1} & v_{1} & 1 & 0 & 0 & 0 & -u_{1} u_{2} & -u_{1} v_{2} & -u_{1} \\
0 & 0 & 0 & u_{2} & v_{2} & 1 & -v_{1} u_{2} & -v_{1} v_{2} & -v_{1}
\end{array}\right] .
$$

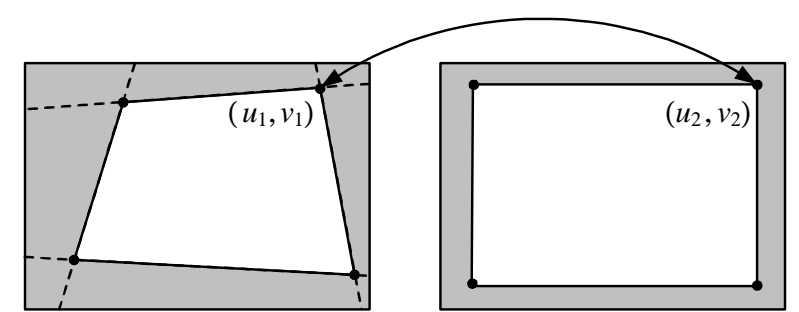

Fig. 1. Image with projective distortion (left) and desired image without distortion (right); the dashed line shows the straight lines that can be found by the Hough or Radon transform.

The pseudosolution of (2) is the last column-vector of matrix $\mathbf{V}$, corresponding to minimal singular value $\Sigma_{\min }$ of singular value decomposition product [10]:

$$
\mathbf{T}_{2 n \times 9}=\mathbf{U} \boldsymbol{\Sigma} \mathbf{V}^{\mathrm{T}}
$$

Algorithms based on a priori information about the form of objects are mainly used to eliminate projective distortions when shooting text sheets or rectangular planar patterns $[5,6]$.

\section{B. Homography Matrix Estimation by means of the Information about the Camera's Angular Orientation and Distance to the View Plane}

Another approach to the projective distortion compensation is a bird's eye view transformation that is used at low-altitude photography. Bird's-eye view technique displays the rectified image of surrounding objects like an "overhead" or "look-down" view [13-15].

The basic idea of a bird's eye view transformation technique is to place the virtual camera (VC) $[8,9,16]$ so that its optical axis is collinear with the normal to the viewed plane and then to estimate the homography matrix between real and virtual cameras.

The geometric formulation of the correction problem using $\mathrm{VC}$ is shown at Fig. 2 (it is assumed that there the camera lens have neither radial nor tangential distortion). In Fig. 2, the real camera $(\mathrm{RC})$ is located at point $\mathbf{C} 1$ with the altitude above view plane $V_{1}$, and the $\mathrm{VC}$ is located at point $\mathbf{C}_{2}$ with height $V_{2}$. Let us suppose also that the point of the $\mathrm{RC}$ and $\mathrm{VC}$ principal axes intersection lies on the observed plane with normal $\mathbf{n}$.

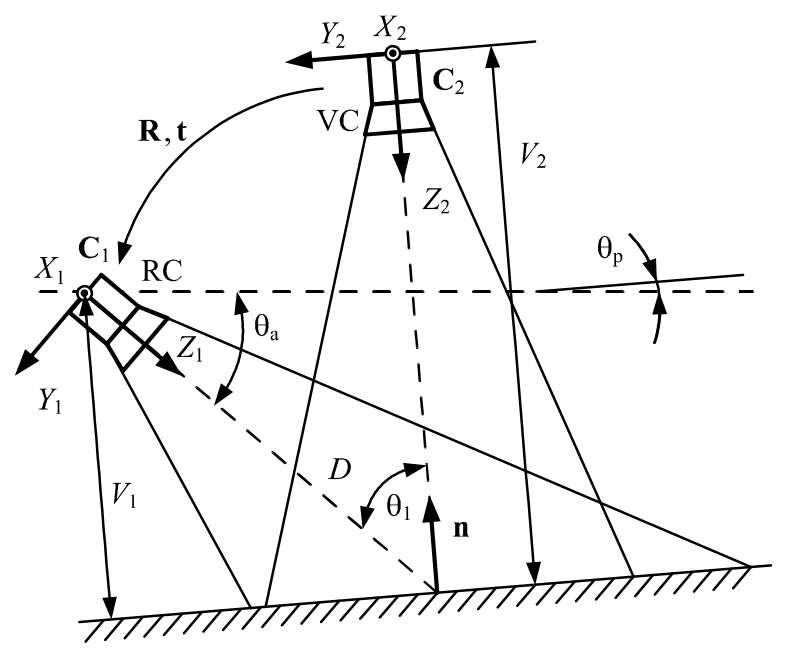

Fig. 2. Mutual position of real and virtual cameras.

The homography matrix for known intrinsic and extrinsic parameters of a pair of projective cameras and the observation of a plane is determined by expression $[10,17]$ :

$$
\mathbf{H}_{\mathrm{VC} \_ \text {to_RC }}=\mathbf{K}_{\mathrm{RC}}\left[\mathbf{R}-\left(\mathbf{t n}^{\mathbf{T}}\right) / d\right] \mathbf{K}_{\mathrm{VC}}{ }^{-1}
$$

where $\mathbf{K}_{\mathrm{RC}}$ and $\mathbf{K}_{\mathrm{VC}}$ are intrinsic matrices of $\mathrm{RC}$ and $\mathrm{VC}$; $d$ is a distance to the observed plane; and $\mathbf{n}$ is a normal vector (according to the geometric constructions in Fig. 2, in the VC coordinate system the following is true: $d=V_{2}$, $\left.\mathbf{n}=[0,0,-1]^{\mathrm{T}}\right)$.

The angular orientation of the $\mathrm{RC}$ relative to the horizon plane (in Fig. 2, it is shown in dashed lines) can be estimated using a pre-calibrated three-axis MEMS accelerometer that is fixed on the camera body. If $X$-axis of the accelerometer is directed along the RC principal axis, $Y$-axis is directed to the left side and $Z$-axis complements its coordinate system for right-handed use, then roll and pitch angles can be estimated as:

$$
\varphi_{\mathrm{a}}=-\operatorname{atan} 2\left(a_{y}, a_{z}\right), \quad \theta_{\mathrm{a}}=-\operatorname{atan} 2\left[a_{x},\left(a_{y}^{2}+a_{z}^{2}\right)^{0.5}\right],
$$

where $\left[a_{x}, a_{y}, a_{z}\right]^{\mathrm{T}}$ is the output signal of the accelerometer taking into account its calibration coefficients [18-20]. By these angles, the rotation quaternion can be calculated [21]:

$$
\mathbf{q}_{\mathrm{a}}=\left[c_{\theta} c_{\varphi}, c_{\theta} s_{\varphi}, s_{\theta} s_{\varphi}, s_{\theta} c_{\varphi}\right]^{\mathrm{T}},
$$

where $c_{\theta}=\cos \left(0.5 \theta_{\mathrm{a}}\right), \quad s_{\theta}=\sin \left(0.5 \theta_{\mathrm{a}}\right), \quad c_{\varphi}=\cos \left(0.5 \varphi_{\mathrm{a}}\right)$, $s_{\varphi}=\sin \left(0.5 \varphi_{\mathrm{a}}\right)$. 
Then, from Fig. 2, the rotation quaternion of the RC coordinate system relative to the $\mathrm{VC}$ coordinate system is:

$$
\mathbf{q}=\mathbf{q}_{\mathrm{vC}}{ }^{*} \bullet \mathbf{q}_{\mathrm{p}}{ }^{*} \bullet \mathbf{q}_{\mathrm{a}},
$$

In formula (5), $\mathbf{q}_{\mathrm{p}}$ is the rotation quaternion of observed plane normal vector $\mathbf{n}$ relative to the horizon plane, and the rotation quaternion of the $\mathrm{VC}$ coordinate system is:

$$
\mathbf{q}_{\mathrm{vC}}=[c, 0,0, s]^{\mathrm{T}}=[1 / \sqrt{2}, 0,0,-1 / \sqrt{2}]^{\mathrm{T}},
$$

where $c=\cos \left(0.5 \theta_{\mathrm{VC}}\right), s=\sin \left(0.5 \theta_{\mathrm{VC}}\right), \theta_{\mathrm{VC}}=-\pi / 2$, and “ $\bullet$ " and " " " are quaternion multiplication and conjugation operators, respectively [21].

To overcome a priori uncertainty about the rotation quaternion of observed plane $\mathbf{q}_{\mathrm{p}}$, the following two methods are possible.

The first method consists in acceptance of a hypothesis regarding the parallelism of observed and horizon planes; and therefore:

$$
\mathbf{q}_{\mathrm{p}}=[1,0,0,0]^{\mathrm{T}} .
$$

The second method can be applied when photo or video shooting is performed from a land vehicle (for example, in case of road lane lines monitoring) and consists in mounting an auxiliary accelerometer on it. Then one approximately can assume that:

$$
\mathbf{q}_{\mathrm{p}}=\left[c_{\theta}{ }^{\text {aux }} c_{\varphi}{ }^{\text {aux }}, c_{\theta}{ }^{\text {aux }} s_{\varphi}{ }^{\text {aux }}, s_{\theta}{ }^{\text {aux }} s_{\varphi}{ }^{\text {aux }}, s_{\theta}{ }^{\text {aux }} c_{\varphi}{ }^{\text {aux }}\right]^{\mathrm{T}},
$$

where $\quad c_{\theta}^{\text {aux }}=\cos \left(0.5 \theta_{\mathrm{a}}^{\text {aux }}\right), \quad s_{\theta}^{\text {aux }}=\sin \left(0.5 \theta_{\mathrm{a}}^{\text {aux }}\right)$, $c_{\varphi}{ }^{\text {aux }}=\cos \left(0.5 \varphi_{\mathrm{a}}{ }^{\text {aux }}\right), s_{\varphi}{ }^{\text {aux }}=\sin \left(0.5 \varphi_{\mathrm{a}}{ }^{\text {aux }}\right)$, and $\theta_{\mathrm{a}}^{\text {aux }}$ and $\varphi_{\mathrm{a}}{ }^{\text {aux }}$ are, respectively, pitch and roll angles estimated by auxiliary accelerometer by formulas (4).

In accordance with the formulas for Rodriguez - Hamilton parameters, quaternion can be transformed to the rotation matrix [21]:

$$
\mathbf{R}=\left[\begin{array}{ccc}
1-2 q_{y}{ }^{2}-2 q_{z}{ }^{2} & 2\left(q_{x} q_{y}-q_{w} q_{z}\right) & 2\left(q_{x} q_{z}+q_{w} q_{y}\right) \\
2\left(q_{x} q_{y}+q_{w} q_{z}\right) & 1-2 q_{x}{ }^{2}-2 q_{z}{ }^{2} & 2\left(q_{y} q_{z}-q_{w} q_{x}\right) \\
2\left(q_{x} q_{z}-q_{w} q_{y}\right) & 2\left(q_{y} q_{z}+q_{w} q_{x}\right) & 1-2 q_{x}{ }^{2}-2 q_{y}{ }^{2}
\end{array}\right]
$$

where $q_{w}$ and $\left[q_{x}, q_{y}, q_{z}\right]^{\mathrm{T}}$ are respectively scalar and vector parts of quaternion.

The coordinates of the RC coordinate system origin in the VC coordinate system are determined from the geometric constructions of Fig. 2:

$$
\mathbf{C}_{1}=\left[0, V_{1} \operatorname{tg} \theta_{1}, V_{2}-V_{1}\right]^{\mathrm{T}},
$$

where $\theta_{1}=\operatorname{atan} 2\left(q_{w} q_{x}-q_{y} q_{z}, q_{w}{ }^{2}+q_{y}{ }^{2}-0,5\right)$.
To estimate the height of the RC relative to the observed plane, either an altimeter or a range meter can be used. In the latter case, altitude $V_{1}$ is estimated from range meter measuring value $D$ :

$$
V_{1}=D \cos \theta_{1} .
$$

By estimated $\mathbf{R}$ and $\mathbf{C}_{1}$, the camera translation vector is calculated for the substitution in (3):

$$
\mathbf{t}=-\mathbf{R C}_{1}
$$

The intrinsic VC matrix

$$
\mathbf{K}_{\mathrm{VC}}=\left[\begin{array}{ccc}
f_{u} & 0 & 0.5 W_{\mathrm{VC}} \\
0 & f_{v} & 0.5 H_{\mathrm{VC}} \\
0 & 0 & 1
\end{array}\right]
$$

can be varied by the values diagonal elements $f_{u}$ and $f_{v}$ for scaling the corrected image; $W_{\mathrm{VC}}$ and $H_{\mathrm{VC}}$ are the width and height of the $\mathrm{VC}$ frame, respectively.

For high quality projective distortion correction, it is desirable to minimize measurement errors of auxiliary sensors (in this work there is a three-axis MEMS accelerometer and an ultrasonic range meter), which is achieved due to their preliminary calibration [18-20].

\section{CAMERA AND AuXILIARY Sensors CALIBRATION}

\section{A. Camera Calibration}

For camera calibration, it is convenient to use the known Zhang calibration algorithm [22] and standard OpenCV libraries [23] or toolboxes like [24] for intrinsic camera parameters matrix and lens distortion coefficients estimation. In this work, 15 test frames of "chessboard" pattern with $9 \times 6$ cells and side of $3 \mathrm{~cm}$ were used.

\section{B. Triaxial MEMS Accelerometer Calibration}

The linear error model of the three-axis MEMS accelerometer is given in [18-20]:

$$
\mathbf{a}_{i}=\mathbf{K}_{\mathrm{a}} \mathbf{T}^{-1} \mathbf{g}_{i}+\mathbf{b}+\mathbf{n}_{\mathrm{a} i}
$$

where $\mathbf{a}_{i}=\left[a_{x i}, a_{y i}, a_{z i}\right]^{\mathrm{T}}$ is an accelerometer output vector at $i$-th sample, $\mathbf{K}_{\mathrm{a}}=\operatorname{Diag}\left\{k_{x}, k_{y}, k_{z}\right\}$ is a diagonal matrix of scale factors, $\mathbf{T}$ is an inter-axis upper triangular misalignment correction matrix;

$$
\mathbf{T}=\left\|\begin{array}{ccc}
1 & -\alpha_{y z} & \alpha_{z y} \\
0 & 1 & -\alpha_{z x} \\
0 & 0 & 1
\end{array}\right\|,
$$


$\alpha_{y z}, \quad \alpha_{z x}, \quad$ and $\alpha_{z y}$ are misalignment coefficients, $\mathbf{g}_{i}=\left[g_{x i}, g_{y i}, g_{z i}\right]^{\mathrm{T}}$ is a $\mathrm{g}$-force vector in platform coordinate at $i$-th sample, $\mathbf{b}=\left[b_{x}, b_{v}, b_{z}\right]^{\mathrm{T}}$ is a zero bias vector of each axis, and $\mathbf{n}_{\mathrm{a} i}=\left[n_{\mathrm{ax}}, n_{\mathrm{a} y}, n_{\mathrm{a} z}\right]^{\mathrm{T}}$ is an accelerometer noise vector at $i$-th sample.

Six or twelve static known accelerometer positions are used for estimation unknown parameters of system of linear equations (8) by finding its pseudosolution [20].

\section{Correction of Ultrasonic Range Meter Measurements}

The speed of sound propagation in air $(\mathrm{m} / \mathrm{s})$ depends on temperature $t$ (in degrees Celsius), pressure $P$ (in hectopascals) and relative humidity $R H$ (in percents) [25-27]:

$$
c_{\mathrm{s}}(t, P, R H)=K_{w}(h) c(t)=K_{w}(h)(331.3+0.6 t),
$$

where the coefficient $K_{w}(h)$ takes into account the difference in sound velocities in dry and moist air,

$$
K_{w}(h)=4.5513[\gamma(h) / M(h)]^{0.5},
$$

$M(h)$ is the molar mass of moist air,

$$
M(h)=M-\left(M-M_{w}\right) h=29-(29-18) h=29-11 h .
$$

$M=29$ is a molar mass of dry air, $M_{w}=18$ is a molar mass of water, parameter

$$
h=0,01 R H e_{\mathrm{p}}(t) / P_{\mathrm{a}},
$$

where $P_{\mathrm{a}}$ is an atmospheric pressure, $e_{\mathrm{p}}(t)$ is a partial pressure of water vapor in moist air, depending on temperature and atmospheric pressure,

$$
e_{\mathrm{p}}(t)=e_{\mathrm{w}}(t) f(P)
$$

where $f(P)=1.0016+3.15 \cdot 10^{-6} P-0.074 P^{-1}$ and $e_{\mathrm{w}}(t)=6.112 \exp [17.62 t /(243.12+t)]$.

In general, the speed of sound propagation in air in addition to temperature, pressure and relative humidity depends on its frequency, however, as shown in [28], for ultrasonic frequencies above $30 \mathrm{kHz}$ this dependence can be neglected.

\section{OBJECT Size Estimation}

If the principal axis of the $\mathrm{VC}$ is oriented normally to the observed plane, then the solution of the problem of measuring the linear dimensions of objects can be found from the condition for the similarity of the triangles formed by points on the object contour and their projections onto the principal plane (Fig. 3): since the triangles $\Delta \mathbf{C c m}_{i}$ and $\Delta \mathbf{C C}_{\mathrm{p}} \mathbf{M}_{i}$ are similar, then

$$
\mathbf{M}_{i}=V \mathbf{m}_{\mathrm{VCin}},
$$

where $V$ is $\mathrm{VC}$ altitude, $\mathbf{C}-\mathrm{VC}$ origin, $\mathbf{c}-\mathrm{VC}$ principal point, $\mathbf{m}_{\mathrm{VC} i}-$ projection of point $\mathbf{M}_{i}$ to the VC principal plane, and $\mathbf{m}_{\mathrm{VCn} i}-$ normalized pixel coordinates of $\mathbf{m}_{\mathrm{VC} i}$,

$$
\mathbf{m}_{\mathrm{VCn} i}=\mathbf{K}_{\mathrm{VC}}{ }^{-1} \mathbf{m}_{\mathrm{VC} i} \text {. }
$$

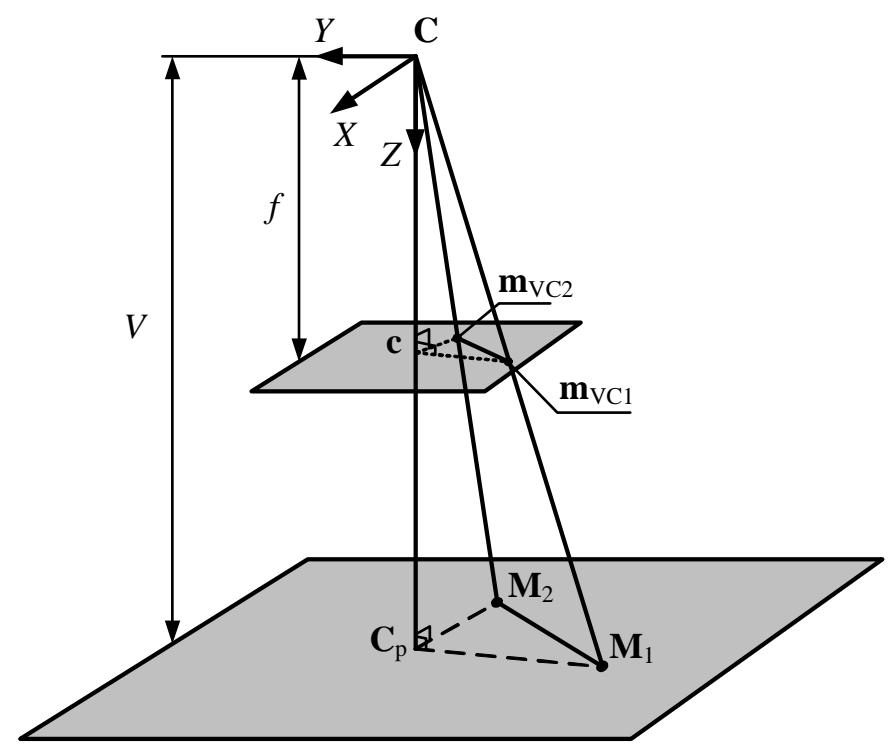

Fig. 3. Explanatory figure to the object size estimation.

The distance between points $\mathbf{M}_{i}$ and $\mathbf{M}_{j}$ is:

$$
\begin{gathered}
d_{i j}=\left\|\mathbf{M}_{i}-\mathbf{M}_{j}\right\|=V \mathbf{K}_{\mathrm{VC}}{ }^{-1}\left\|\mathbf{m}_{\mathrm{VC} i}-\mathbf{m}_{\mathrm{VC} j}\right\|= \\
V\left[\mathbf{R}-\left(\mathbf{t n}^{\mathbf{T}}\right) / d\right]^{-1} \mathbf{K}_{\mathrm{RC}}{ }^{-1}\left\|\mathbf{m}_{\mathrm{RC} i}-\mathbf{m}_{\mathrm{RC} j}\right\| .
\end{gathered}
$$

If the object contour is triangle (t) or quadrilateral (q), then its area can be estimated by formulas

$$
S_{\mathrm{t}}=\left[s\left(s-d_{12}\right)\left(s-d_{13}\right)\left(s-d_{23}\right)\right]^{0.5}
$$

and

$$
\begin{gathered}
S_{\mathrm{q}}=\left[\left(s-d_{12}\right)\left(s-d_{23}\right)\left(s-d_{34}\right)\left(s-d_{41}\right)-\right. \\
\left.-0.25\left(d_{12} d_{34}+d_{23} d_{41}+p q\right)\left(d_{12} d_{34}+d_{23} d_{41}-p q\right)\right]^{0.5},
\end{gathered}
$$

respectively, where $d_{i j}$ is the length of the side between vertexes with numbers $i$ and $j ; s$ is the semiperimeter; and $p$ and $q$ are quadrilateral diagonals.

Additionally, for wide-angle cameras, it is necessary to correct the distortion of their lenses according to the Brown Conrady model [29] and to solve the system of equations:

$$
u_{\mathrm{n} d}=u_{\mathrm{n}}\left[1+k_{1} r^{2}+k_{2} r^{4}+k_{3} r^{6}\right]+\left[p_{1}\left(r^{2}+2 x_{\mathrm{H}}^{2}\right)+2 p_{2} x_{\mathrm{H}} y_{\mathrm{H}}\right],
$$

$$
v_{\mathrm{n} d}=v_{\mathrm{n}}\left[1+k_{1} r^{2}+k_{2} r^{4}+k_{3} r^{6}\right]+\left[2 p_{1} x_{\mathrm{H}} y_{\mathrm{H}}+p_{2}\left(r^{2}+2 y_{\mathrm{H}}^{2}\right)\right],
$$


where $k_{i}, i=1 . .3$, is the radial distortion coefficients; $p_{i}$, $i=1,2$, are the tangential distortion coefficients; and $\left(u_{\mathrm{n} d i}, v_{\mathrm{n} d i}\right)$ and $\left(u_{\mathrm{n} i}, v_{\mathrm{n} i}\right)$ are normalized pixel coordinates of RC with distortion and without it, respectively, $\left[u_{\mathrm{n}}, v_{\mathrm{n}}, 1\right]^{\mathrm{T}}=\mathbf{K}_{\mathrm{RC}}{ }^{-1}[u, v, 1]^{\mathrm{T}}, r=\left(u_{\mathrm{n}}{ }^{2}+v_{\mathrm{n}}{ }^{2}\right)^{0.5}$.

\section{EXPERIMENTS}

\section{A. Model Experiments}

The influence of errors in the measurement of angular coordinates was investigated by the simulation method in Mathcad: the matrices of RC and VC internal parameters were set $\mathbf{K}_{\mathrm{RC}}=\left[\begin{array}{ccc}900 & 0 & 640 \\ 0 & 900 & 360 \\ 0 & 0 & 1\end{array}\right]$ and $\mathbf{K}_{\mathrm{VC}}=\left[\begin{array}{ccc}3375 & 0 & 640 \\ 0 & 3375 & 360 \\ 0 & 0 & 1\end{array}\right]$. Altitudes of RC and VC were set $H_{1}=800 \mathrm{~mm}$ and $H_{2}=3000 \mathrm{~mm}$; angular orientation of observed plane and camera were set $\theta_{\mathrm{p}}=\psi_{\mathrm{p}}=0$ and $\theta_{\mathrm{a}}=\pi / 4, \psi_{\mathrm{a}}=-\pi / 6$, respectively; coordinates of observed object (square with side length $L=500 \mathrm{~mm}$ ) in the VC coordinate system were set $\mathbf{M}_{1}=\left[\begin{array}{lll}-0,5 L, & -0,5 L, & V_{2}\end{array}\right]^{\mathrm{T}}, \quad \mathbf{M}_{2}=\left[\begin{array}{lll}0,5 L, & -0,5 L, & V_{2}\end{array}\right]^{\mathrm{T}}$, $\mathbf{M}_{3}=\left[-0,5 L, 0,5 L, V_{2}\right]^{\mathrm{T}}, \mathbf{M}_{4}=\left[0,5 L, 0,5 L, V_{2}\right]^{\mathrm{T}}$.

To simulate measurement errors of the three-axis MEMS accelerometer, the normalized value of the bias offset $b_{\mathrm{n}}$ for each of its sensitivity axes in (8) was added.

The pixel coordinates of the square corners on the images of the $\mathrm{RC} \mathbf{m}_{\mathrm{RC} i}$ and $\mathrm{VC} \mathbf{m}_{\mathrm{VC} i}$ with considering (3)-(7) were calculated by formulas:

$$
\begin{gathered}
\mathbf{m}_{\mathrm{RC} i}=w_{i}^{-1} \mathbf{K}_{\mathrm{RC}}\left[\mathbf{R} \mathbf{M}_{i}+\mathbf{t}\right], \\
\mathbf{m}_{\mathrm{VC} i}=\mathbf{K}_{\mathrm{VC}} \mathbf{M}_{i},
\end{gathered}
$$

where parameter $w_{i}$ is the depth of point $\mathbf{M}_{i}$ from the $\mathrm{RC}$ center in the direction of its principal ray [10].

The simulation results are shown in Table 1 and Fig. 4. An area of square was calculated by formula (12).

TABLE I. MOdELling OF THE SQUARE AREA Estimation

\begin{tabular}{|l|c|c|c|c|c|}
\hline $\begin{array}{l}\text { Additive accelerometer } \\
\text { bias error, } b_{\mathrm{n}} \times 10^{3}\end{array}$ & 1 & 2 & 3 & 4 & 5 \\
\hline $\begin{array}{l}\text { Angle estimation error, } \\
\text { degree }\end{array}$ & 0.57 & 1.15 & 1.72 & 2.3 & 2.86 \\
\hline $\begin{array}{l}\text { Area estimation relative } \\
\text { error, } \%\end{array}$ & 3 & 6.3 & 9.75 & 13.4 & 17.1 \\
\hline
\end{tabular}




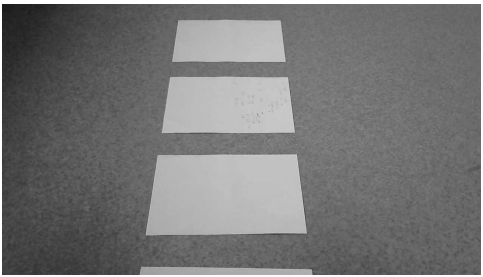

a)

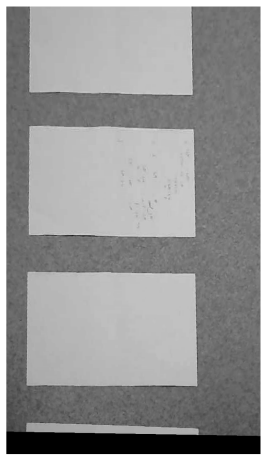

b)
Fig. 5. The results of implementation of bird's eye view transormation technique into the webcam image (A4 sheets): $\theta_{\mathrm{a}}=-46.1^{\circ}$, $\varphi_{\mathrm{a}}=-1.2^{\circ}, V_{1}=860 \mathrm{~mm}, V_{2}=2000 \mathrm{~mm}, \theta_{\mathrm{p}}=\psi_{\mathrm{p}}=0$, VC zoom is $4 \mathrm{x}$ : a) Logitech $\mathrm{C} 270$ frame, b) VC frame

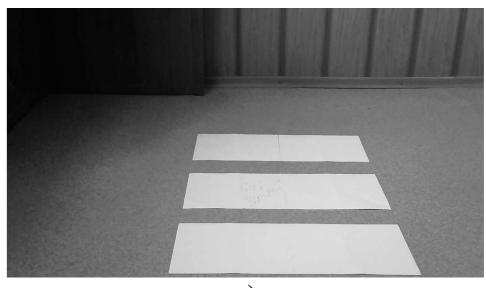

a)

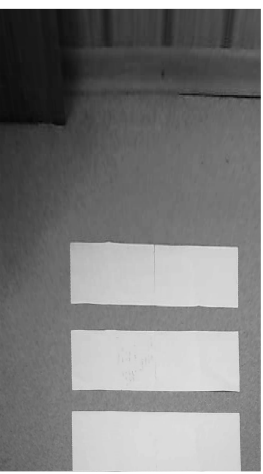

b)
Fig. 6. The results of implementation of bird's eye view transormation technique into the webcam image (two docked A4 sheets): $\theta_{\mathrm{a}}=-25.2^{\circ}, \varphi_{\mathrm{a}}=$ $0^{\circ}, V_{1}=852 \mathrm{~mm}, V_{2}=2000 \mathrm{~mm}, \theta_{\mathrm{p}}=\psi_{\mathrm{p}}=0$, VC zoom is $2 \mathrm{x}$ : a) Logitech $\mathrm{C} 270$ frame, b) VC frame

TABLE II. QUADRILATERAL AREA ESTIMATION RESULTS

\begin{tabular}{|l|c|c|}
\hline Input image & Fig. 5, b & Fig. 6, b \\
\hline Estimation of area, $\mathrm{cm}^{2}$ & 595.282 & $1,183.87$ \\
\hline Area estimation relative error, \% & 4.6 & 5.1 \\
\hline
\end{tabular}

\section{CONCLUSION}

For a low-altitude photography, the use of the bird's eye view transformation algorithm for correcting projective distortions by means of a virtual camera and auxiliary sensors (a three-axis accelerometer and altimeter) makes it possible to estimate the area of planar objects with a relative error that is no more than $5 \%$.

\section{References}

[1] D. Rocchini, M. Metz, A. Frigeri, L. Delucchi, M. Marcantonio, and M. Neteler, "Robust rectification of aerial photographs in an open source environment", Computers \& Geosciences, vol. 39, pp. 145-151, 2012.

[2] V.A. Fursov, Ye.V. Goshin, and A.P. Kotov, "The hybrid CPU/GPU implementation of the computational procedure for digital terrain models generation from satellite images", Computer Optics, vol. 40, no. 5 , pp. $721-728,2016$.
[3] J.-I. Jung and Y.-S. Ho, "Geometric and colorimetric error compensation for multi-view images”, J. Vis. Commun. Image, vol. 25, pp. 698-708, 2014.

[4] Y. Kang and Y. Ho, "An efficient image rectification method for parallel multi-camera arrangement", IEEE Trans. on Consumer Electronics, vol. 57, no. 3, pp. 1041-1048, 2011.

[5] L. Jagannathan and C. V. Jawahar, "Perspective correction methods for camera based document analysis", Camera-based Document Analysis and Recognition, pp. 148-154, August 2005 [First Int. Workshop on Camera-based Document Analysis and Recognition, p. 164, 2005].

[6] X. Wang, R. Klette, and B. Rosenhahn, "Geometric and photometric correction of projected rectangular pictures", Proc. of Image Vision Comput. New Zealand, pp. 223-228, November 2005 [Image Vision Comput. New Zealand, p. 510, 2005].

[7] Y.-C. Liu, K.-Y. Lin, and Y.-S. Chen, "Bird's-eye view vision system for vehicle surrounding monitoring", Robot vision, pp. 207-218, February 2008. [2nd Int. Workshop on Robot Vision "RobVis 2008", p. 468,2008$]$.

[8] C.-C. Lin and M.-S. Wang, "A vision based top-view transformation model for a vehicle parking assistant", Sensors, vol. 12, no. 4, pp. 4431-4446, 2012.

[9] M. Li, C. Zhao, Y. Hou, and M. Ren, "A new lane line segmentation and detection method based on inverse perspective mapping", Int. J. of digital content technology and its applications, vol. 5, no. 4, pp. 230-236, 2011.

[10] R. Hartley and A. Zisserman, Multiple view geometry in computer vision, 2nd ed. Cambridge: Cambridge University Press, 2003, pp. 87117.

[11] G. Xu and Z. Zhang, Epipolar geometry in stereo, motion and object recognition. A unified approach. Dordrecht: Kluwer Academic Publishers, 1996, pp. 26-41.

[12] R.C. Gonzalez, R.E. Woods, Digital image processing, 2nd ed. London: Pearson Education Ltd., 2001, pp. 587-591.

[13] Q.-F. Zhou, J.-H. Liu, X. Wang, and M.-C. Sun, "Automatic correction of geometric distortion in aerial zoom squint imaging", Optics and Precision Engineering, vol. 23, no. 10, pp. 2927-2942, 2015.

[14] M. Venkatesh and P. Vijayakumar, "A simple bird's eye view transformation technique", Int. J. of Scientific and Engineering Research, vol. 3, is. 5, pp. 735-738, 2012.

[15] R. Laganiere, "Compositing a Bird's eye view mosaic", Proc. of Vision Interface'2000, pp. 382-387, May 2000 [13th Canadian Conf. Vision Interface, p. 408, 2000].

[16] Z. Jia, A. Gallagher, and T. Chen, "Cameras and gravity: estimating planar object orientation", Proc. of IEEE Int. Conf. on Image Processing, pp. 3642-3646, September 2013 [20th IEEE Int. Conf. on Image Processing, p. 4592, 2013].

[17] T. Luhmann, S. Robson, S. Kyle, and J. Boehm, Close-range photogrammetry and 3D imaging. Berlin: De Gruyter, 2013.

[18] J.K. Bekkeng, "Calibration of a novel MEMS inertial reference unit", IEEE Trans. Instrum. Meas, vol. 58, pp. 1967-1974, 2009.

[19] L. Wang and F. Wang, "Intelligent calibration method of low cost MEMS inertial measurement unit for an FPGA-based navigation system," International J. of Intelligent Engineering and Systems, vol. 4, no. 2, pp. 32-41, 2011.

[20] J. Yang, W. Wu, Y. Wu, and J. Lian, "Improved iterative calibration for triaxial accelerometers based on the optimal observation", Sensors, vol. 12, no. 6, pp. 8157-8175, 2012.

[21] J.B. Kuipers, Quaternions and rotation sequences: a primer with applications to orbits, aerospace, and virtual reality. New Jersey: Princeton University, 1998, pp. 155-176.

[22] Z. Zhang, "A flexible new technique for camera calibration," IEEE Trans. on Pattern Analysis and Machine Intelligence, vol. 22, no. 11, pp. 1330-1334, 2000.

[23] Camera Calibration and 3D reconstruction, Web reference: http://docs.opencv.org/2.4/modules/calib3d/doc/camera_calibration_and_3d reconstruction.html.

[24] Camera Calibration Toolbox for Matlab, Web reference: http://www.vision.caltech.edu/bouguetj/calib_doc/ 
[25] D.A. Bohn, "Environmental effects on the speed of sound", J. Audio Eng. Soc., vol. 36, no. 4, pp. 223-231, 1988.

[26] T. Löfqvist, K. Sokas, and J. Delsing, "Speed of sound measurements in humid air using an ultrasonic flow meter", Metrology in the 3rd Millennium, pp. 1650-1653, June 2003 [17th IMEKO World Congress, 1 CD-ROM, 2003].

[27] W. Van Schaik, M. Grooten, T. Wernaart, and C. Van der Geld, "High accuracy acoustic relative humidity measurement induct flow with air", Sensors, vol. 10, no. 8, pp. 7421-7433, 2010 .
[28] G.P. Howell and C.L. Morfey, "Frequency depence of the speed of sound in air", J. Acoust. Soc. Am., vol. 82, no. 1, pp. 375-376, 1987.

[29] D.C. Brown, "Close-range camera calibration", Photogrammetric engineering, vol. 37 , no. 8, pp. 855-866, 1971.

[30] C. Harris and M. Stephens, "A combined corner and edge detector", Proc. of 4th Alvey Vision Conf., pp. 147-151, August-September 1988. [4th Alvey Vision Conf., p. 302, 1988]. 